\title{
The role of amino acid-induced mammalian target of rapamycin complex 1 (mTORC1) signaling in insulin resistance
}

\author{
Mee-Sup Yoon ${ }^{1}$ and Cheol Soo Choi ${ }^{1,2,3}$ \\ Mammalian target of rapamycin (mTOR) controls cell growth and metabolism in response to nutrients, energy, and growth \\ factors. Recent findings have placed the lysosome at the core of mTOR complex 1 (mTORC1) regulation by amino acids. Two \\ parallel pathways, Rag GTPase-Ragulator and Vps34-phospholipase D1 (PLD1), regulate mTOR activation on the lysosome. This \\ review describes the recent advances in understanding amino acid-induced mTOR signaling with a particular focus on the role of \\ mTOR in insulin resistance.
}

Experimental \& Molecular Medicine (2016) 48, e201; doi:10.1038/emm.2015.93; published online 8 January 2016

\section{INTRODUCTION}

Amino acids act as nutrient signals to induce cell growth in addition to their role as protein building blocks. Cells can sense the availability of extracellular and intracellular amino acids and initiate multiple biochemical processes to synthesize new cellular components. Starvation and low energy status initiate an alternate set of processes that suppress biosynthetic programs and increase internal sources of metabolites by recycling aged proteins and organelles. Moreover, prolonged starvation and hypoglycemia induce the catabolism of amino acids to produce other forms of energy such as glucose and ketone bodies. Therefore, accurate amino acid sensing is crucial for the efficient control of cellular metabolism.

The mammalian target of rapamycin (mTOR) signaling pathway has a central role in amino acid sensing and signaling. mTOR is a serine/threonine kinase that functions as a nutrient sensor as well as a growth regulator. In this minireview, we begin by summarizing the current understanding of amino acid-induced mTOR complex1 (mTORC1) regulation. We then discuss how mTORC1 activation by amino acids controls insulin signaling, a key aspect of body metabolism, and how deregulation of mTOR signaling can promote metabolic disease.

\section{ORGANIZATION OF MTOR COMPLEXES}

The TOR protein was identified as the target of the immunosuppressant rapamycin by genetic and biochemical approaches in yeast and mammals. ${ }^{1}$ TOR, and its mammalian ortholog mTOR, are members of phosphoinositide-3-kinase-related protein kinases family and control a range of cellular processes in response to nutrient and energy levels, including cell growth, differentiation, proliferation and metabolic homeostasis. ${ }^{1}$ The TOR proteins are unique among phosphoinositide-3-kinaserelated protein kinases family members in that they bind directly to rapamycin through an FKBP12 rapamycin-binding domain. ${ }^{2}$ mTOR exists as part of two biochemically and functionally distinct complexes: mTOR complex 1 (mTORC1) and mTOR complex 2 (mTORC2) (Figure 1). ${ }^{3,4}$ mTORC1 contains regulatory-associated protein of $\mathrm{mTOR}$ (raptor), while mTORC2 contains rapamycin-insensitive companion of mTOR (rictor). These partners act as scaffolds for assembling the complexes and bind substrates and regulators. ${ }^{1}$ mTORC2 also contains protein observed with rictor-1 (Protor-1), Protor-2 and mammalian stress-activated protein kinase-interacting protein 1 (mSIN1). ${ }^{5-7}$ Mammalian lethal with SEC13 protein 8 (mLST8; also known as G $\beta$ L) plays a part in both mTORC1 and mTORC2. ${ }^{8}$ mTOR complexes are also associated with several

\footnotetext{
${ }^{1}$ Department of Molecular Medicine, School of Medicine, Gachon University, Incheon, Korea; ${ }^{2}$ Korea Mouse Metabolic Phenotyping Center (KMMPC), Lee Gil Ya Cancer and Diabetes Institute, Gachon University, Incheon, Korea and ${ }^{3}$ Department of Endocrinology, Internal Medicine, Gachon University Gil Medical Center, Incheon, Korea

Correspondence: Professor M-S Yoon or Professor CS Choi, Department of Molecular Medicine, Lee Gil Ya Cancer and Diabetes Research Instisute, School of Medicine, Gachon University, 7-45 Songdo-Dong, Yeonsu-Gu, Incheon 406-840, Korea.

E-mail: msyoon@gachon.ac.kr or cschoi@gachon.ac.kr

Received 21 July 2015; revised 16 September 2015; accepted 2 October 2015
} 


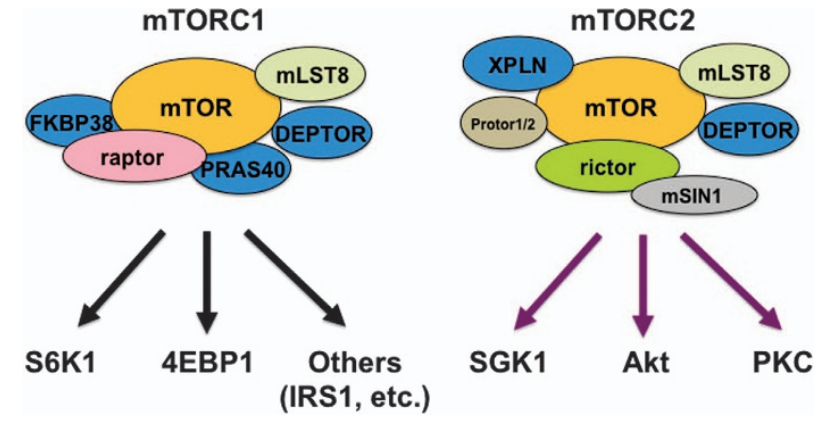

Figure 1 The components of mTORC1 and mTORC2. mTORC1 is comprised of mTOR, raptor and $\mathrm{mLST} 8(\mathrm{G} \beta \mathrm{L})$. In addition, mTORC1 binds to endogenous inhibitors such as PRAS40, DEPTOR and FKBP38. MTORC1 regulates protein translation by phosphorylating S6K1 and 4E-BP1. In contrast, mTORC2 includes mTOR, rictor, $\mathrm{mLST} 8$, protor $1 / 2$ and $\mathrm{mSin} 1$, as well as endogenous inhibitors such as DEPTOR and XPLN. The mTORC2 controls cell survival by regulating SGK, Akt and PKC. Proteins shown in blue are endogenous inhibitors of mTOR.

endogenous inhibitors that regulate their activity, such as proline-rich Akt substrate 40 (PRAS40) and FKBP38 for mTORC1, while exchange factor found in platelet, leukemic and neuronal tissues (XPLN) negatively regulates mTORC2. ${ }^{9-11}$ DEP domain-containing mTOR interacting protein (DEPTOR) binds to both mTORC1 and mTORC2 to control their activities. $^{12}$

It is well known that mTORC1 regulates cell growth in response to several extracellular and intracellular signals including nutrients, mitogens, cellular energy status and various stressors. ${ }^{13}$ The tuberous sclerosis complex 1 (TSC1)TSC2 and Ras homologue enriched in brain (Rheb) have been identified as a major hub for signal transduction components upstream of mTORC1. ${ }^{14-16}$ TSC2 serves as a GTPase-activating protein (GAP) and negative regulator for the small GTPase protein Rheb, which, itself, is a powerful stimulator of mTORC1 kinase activity. ${ }^{17}$ The two most well-known downstream targets of mTORC1 are S6 kinase 1 (S6K1) and eukaryotic translation initiation factor 4E-binding protein 1 (4EBP1), both regulators of protein synthesis. ${ }^{18,19}$

In comparison with mTORC1, the regulation of mTORC2 is poorly established. Only growth factors activate mTORC2 that phosphorylates AGC kinase family members. mTORC2 activates Akt, serum- and glucocorticoid-regulated kinase (SGK), and protein kinase $\mathrm{C}(\mathrm{PKC})$. The regulation of Ser 473-Akt by mTORC2 is especially important since Ser 473 phosphorylation in the hydrophobic motif prepares Akt for further phosphorylation at Thr308 in catalytic domain by phosphoinositidedependent protein kinase 1 (PDK1). ${ }^{1}$ These two phosphorylations induce full activation of Akt that are critical in the pathogenesis of diabetes and cancers.

\section{AMINO ACID SIGNALING}

Of several extracellular and intracellular signals, amino acid availability is considered a crucial mTORC1 activator ${ }^{20,21}$ and the TSC1-TSC2-Rheb axis has long been assumed to transmit amino acid signals to mTOR..$^{22}$ However, mTORC1 signaling in TSC $-/-$ mouse embryonic fibroblasts remains responsive to amino acids, implying the existence of an alternative signaling mechanism. ${ }^{23}$ To date, two other parallel pathways have been found to activate mTORC1 downstream of amino acid stimulation, namely, the Rag-Ragulator and vacuolar protein sorting 34 (Vps34) -phospholipase D1 (PLD1) pathways (Figure 2).

\section{Rag-Ragulator}

A requirement for Rag GTPases in amino acid sensing through mTORC1 was suggested following loss-of-function studies in mammalian, fly and yeast cells. ${ }^{24-26}$ In mammals, there are four members of the Rag subfamily: $\operatorname{RagA}, \operatorname{RagB}, \operatorname{RagC}$ and RagD. The four members function as obligate heterodimers, a feature that is unique among small GTPase subfamilies. An additional layer of unique complexity is that the nucleotidebound state of the Rags is regulated by amino acid sufficiency. During amino acid stimulation, RagA/B binds GTP to form an active Rag complex that recruits raptor at the lysosome, suggesting that Rag is a docking site for mTORC1 at this compartment. Lysosomal targeted mTORC1 made S6K1 phosphorylation insensitive to amino acid starvation, suggesting that the localization of mTORC1 on lysosome is critical for amino acid sensing. ${ }^{27}$ This recruitment is dependent on Ragulator, a Rag-interacting complex, which tethers the Rag GTPases to the lysosome. The tethering function of Ragulator is promoted by myristoylation and palmitoylation on the $\mathrm{N}$-terminus of Ragulator. ${ }^{27}$ Moreover, Ragulator preferentially binds to Rags devoid of nucleotides, and functions as a guanine nucleotide exchange factor (GEF) for RagA/B. ${ }^{28}$ The studies of constitutively active RagA/B proteins suggested that the active GTPbound state of Rag $\mathrm{A} / \mathrm{B}$ proteins is crucial for amino acid sensing and signaling. ${ }^{26}$ Interestingly, the GEF activity of Ragulator is blocked by its interaction with vacuolar $\mathrm{H}^{+}$ ATPase (V-ATPase) at the lysosomal membrane during amino acid starvation. During amino acid stimulation, the GEF activity of Ragulator is fully activated by a conformational change of the V-ATPase-Ragulator complex. ${ }^{29}$ Recently, BarPeled et al..$^{30}$ identified GATOR1, the GAP for RagA/B, which is negatively regulated by GATOR2, even though it is not clear the mechanism how amino acids regulate GATOR2. Inhibition of GATOR1 makes mTORC1 insensitive to amino acid deficiency. The nucleotide state of $\mathrm{RagC} / \mathrm{D}$ also governs the raptor-Rag GTPase interaction. ${ }^{26}$ The tumor suppressor folliculin (FLCN) was identified as a GAP for RagC and RagD, working with its binding partner folliculin-interacting protein 1 (FNIP1) to promote GDP-bound $\mathrm{RagC/D}$ and, thereby, promoting interaction with raptor. ${ }^{31}$ Recently, two independent studies have shown that the tRNA-charging enzyme leucyl-tRNA synthetase (LeuRS) also mediates amino acid signaling upstream of mTORC1. ${ }^{32,33}$ In mammalian cells, LeuRS binds to RagD and functions as a GAP in a leucinedependent manner. However, the GAP activity for RagD was not reproduced in a subsequent study. ${ }^{31}$ Further work is needed to resolve these conflicting data and reconcile these 

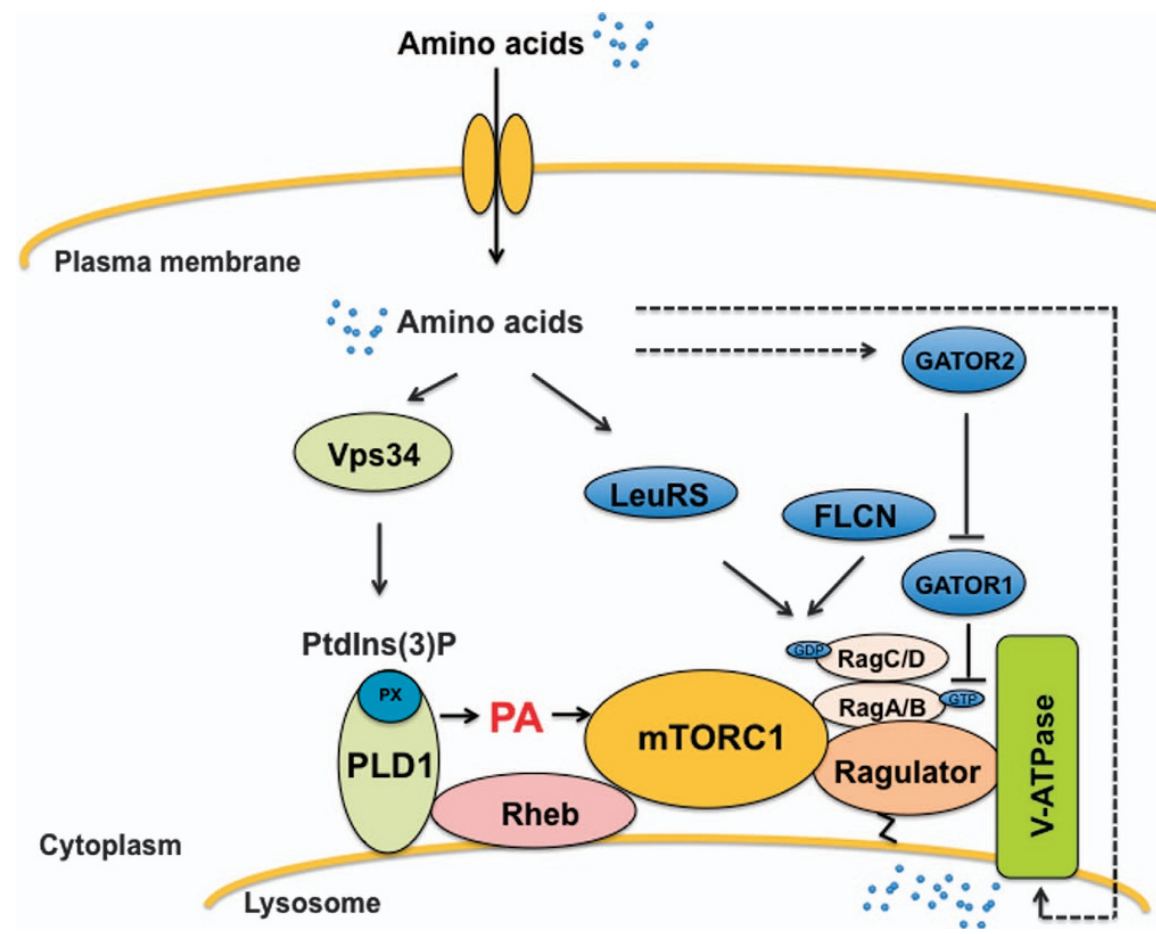

Figure 2 Mechanism by which mTORC1 senses amino acids. Amino acids signal to mTOR through two parallel pathways, Vps34/PLD1 and Rag-Ragulator. Amino acids activate Vps34, which produces Ptdlns(3)P. Ptdlns(3)P mediates the activation of PLD1 and the recruitment of PLD1 to lysosome. The activated PLD1 produces PA on the lysosome. Separate from Vps34/PLD1, amino acids activate Rag GTPases to recruit mTORC1 to the surface of lysosome where mTORC1 binds to Rheb. On the lysosome, mTOR interacts with Rheb and binds to PA. This activates mTORC1 and mediate signaling. GATOR1 functions as a GAP (GTPase-activating protein) for RagGTPase A/B to regulate mTOR negatively. Ragulator also regulates the GTP level of RagGTPase A/B as a GEF (guanine nucleotide exchange factor). The GDP level of Rag GTPase C/D is regulated by FLCN and LeuRS. mTORC1; mTOR complex1, Vps34; class III PI3 kinase, PLD1; phospholipase 1, Rheb; Ras-homologue enriched in brain, PA; phosphatidic acid, FLCN; folliculin, GATOR1; a complex of nitrogen permease regulator 2like protein (NPRL2), NPRL3 and DEP domain-containing protein 5 (DEPDC5), GATOR2; a complex of Mios, SEC13 homologue like 1 (SEH1L), DDB1-and CUL4-associated factor 11(DCAF11:also known as WDR23), WD repeat-containing protein 59 (WDR59) and secretory 13(SEC13).

mechanisms with those of other positive regulators of amino acid signaling.

\section{Vps34-PLD}

Vps34, which was first reported as an amino acid mediator in mTOR signaling, ${ }^{23,34}$ is a class III PI3 kinase that produces phosphatidylinositol 3-phosphate (PtdIns3P) from phosphatidylinositol. ${ }^{35} \mathrm{Vps} 15$, a regulatory subunit of class III PI3 kinase, is required for the stability and activity of Vps34. ${ }^{35}$ During amino acid stimulation, phosphorylation of S6K1 is dampened by siRNA-mediated Vps34 knockdown or by FYVE domain overexpression to sequester PtdIns3P. ${ }^{23}$ The addition of amino acids to starved cells increases Vps34 kinase activity, which is detected by anti-PtdIns3P staining. ${ }^{23}$ These observations suggest that Vps34 serves as an upstream regulator of mTORC1 in amino acid sensing.

The involvement of PLD in amino acid signaling was suggested based on the observation that amino acids are required for mitogen-induced PLD activation. ${ }^{36}$ PLD catalyzes the conversion of phosphatidylcholine to phosphatidic acid (PA), which is a lipid second messenger. ${ }^{37} \mathrm{PA}$ was also found to bind the FRB domain of mTOR, compete with rapamycin and activate mTOR signaling. ${ }^{38}$ Recently, it was shown that PA binding to mTOR activates mTORC1 kinase activity and displaces mTORC1 inhibitors, suggesting a role for PA in mTORC1 regulation. ${ }^{39,40}$ Moreover, PLD1 knockdown impinges on amino acid- and mitogen-induced mTOR activation $^{36,41,42}$ and the availability of amino acids or glucose regulates PLD activity. ${ }^{42,43}$

The mechanism by which Vps34 regulates PLD1/mTOR signaling involves PtdIns3P, the product of Vps34, which binds to the PX domain of PLD1 to regulate amino acid signaling. ${ }^{42}$ The pharmacological inhibition or genetic depletion of Vps34 decreases amino acid-induced PLD activity. In addition, PtdIns3P activates PLD activity in cells expressing wild-type PLD1, but not in cells expressing PX-deleted PLD1, indicating that the PX domain of PLD1 is necessary for amino acidVps34-mTOR signaling. Interestingly, PLD1 translocation to the lysosome, where mTOR resides, is dependent on PtdIns3P and Vps34. The activity and translocation of PLD1 are not affected by Rag, Ragulator or raptor, which control mTOR translocation to the lysosome. These observations suggest that the Vps34-PLD1 pathway acts in parallel with the Rag pathway, 
with both pathways converging at the lysosome to activate mTORC1 signaling.

\section{mTORC1 AND INSULIN RESISTANCE}

Amino acids are well-accepted nutrient signals that have direct or indirect effects at both the cellular and organismal level. Interestingly, circulating levels of branched chain amino acids (BCAAs) are increased in insulin-resistant obesity and type 2 diabetes. ${ }^{44-46}$ In clinical case studies, the increased levels of BCAAs positively correlate with insulin resistance, ${ }^{47}$ and have been reported to be predictive of future insulin resistance or type 2 diabetes in several longitudinal studies of different cohorts. ${ }^{45,48}$ These observations raise the intriguing question of whether increased BCAAs are causal or simply a result of insulin resistance.

mTORC1 activation is a plausible mechanism to explain the link between insulin resistance and BCAAs. mTORC1 hyperactivation gives rise to a negative feedback loop of insulin signaling which negatively regulates insulin signaling. ${ }^{49}$ Active mTORC1 and S6K1 phosphorylate insulin receptor substrate 1 (IRS1), which is an adaptor protein that recruits key downstream effectors to the insulin receptor. ${ }^{50,51}$ Serinephosphorylated IRS1 prompts inactivation of Akt by blocking the recruitment of phosphoinositide-3-kinase to IRS1, leading to insulin desensitization. ${ }^{1}$ mTOR also causes insulin insensitivity by phosphorylating growth factor receptor-bound protein 10 (Grb10). ${ }^{52,53}$ Grb10 reduces tyrosine phosphorylation of insulin receptor normally induced by insulin and IGFs which, in turn, blocks Akt activation..$^{54}$ Phosphorylation of Grb10 by mTORC1 increases Grb10 stability and results in dampened insulin sensitivity. These events translate into reduced glucose uptake and glycogen synthesis in liver and muscles, and increased gluconeogenesis and glucose release by the liver, leading to worsening of hyperglycemia and hyperinsulinemia ${ }^{1}$ (Figure 3 ).

Hyperactivation of mTORC1 is an important paradox in insulin resistance; how can mTORC1 remain constitutively active in an insulin-resistant state if mTORC1 is also activated by insulin? The paradox can be explained if excess nutrients promote hyperactivation of mTORC1 independent of insulin signaling. This is supported by evidence from clinical studies that high levels of BCAAs correlate with insulin resistance. ${ }^{46,55}$ The BCAAs comprise the three essential amino acids having nonlinear aliphatic side chains: leucine, isoleucine and valine, which are more potent activators of mTOR. ${ }^{20,26}$ Becides BCAA, glutamine and arginine have been implicated in mTOR activation, confirming that amino acids activate mTOR..$^{56,57}$ More direct evidence is found in a rat model with high blood levels of amino acids wherein mTORC1 activity is maintained,

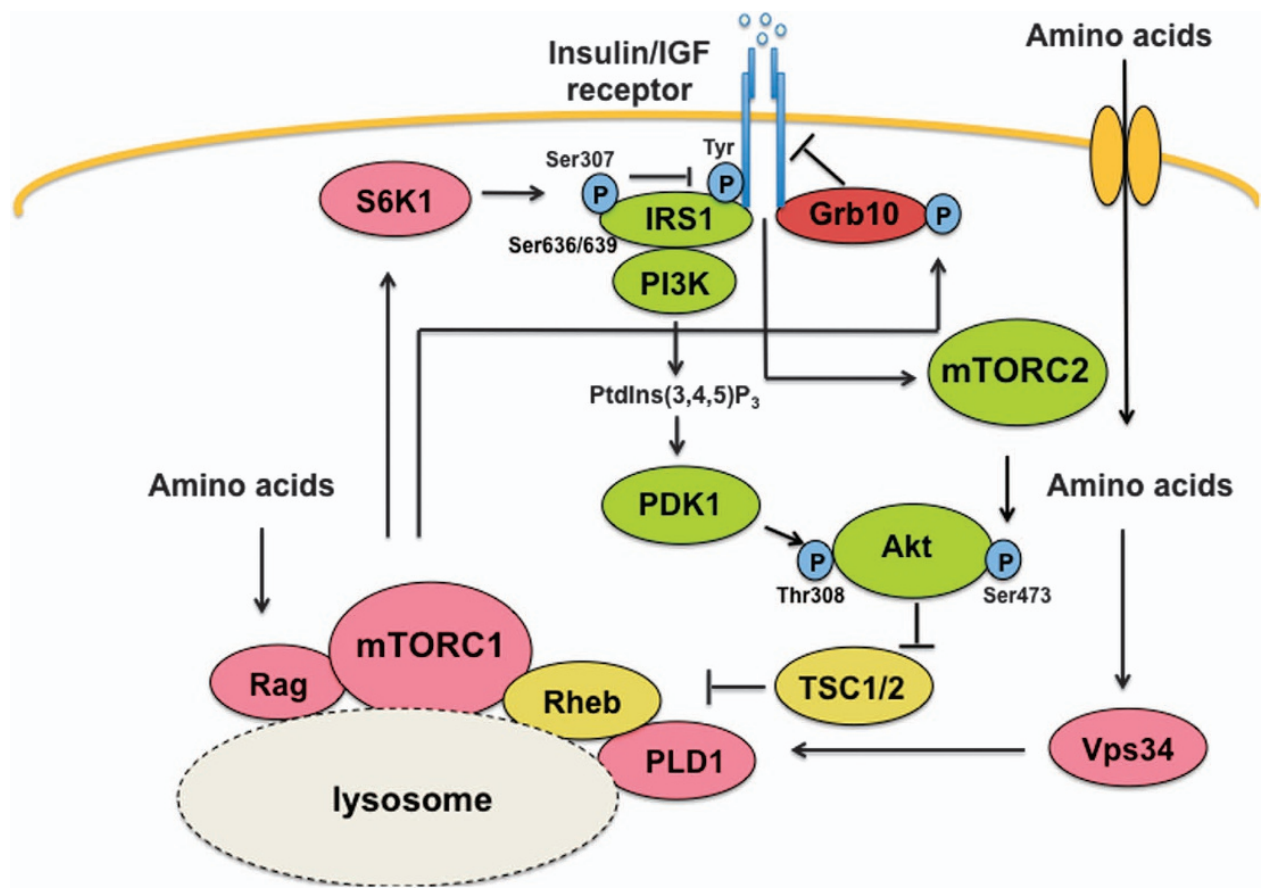

Figure 3 Amino acid-induced mTOR signaling in insulin resistance. Insulin stimulates PI3K to generate phosphatidyl inositol-3,4,5triphosphate(PI(3,4,5,)P3), which promote phosphorylation of Thr308 -Akt by PDK1. Insulin also activates mTORC2 that phosphorylates Akt at Ser 473. Akt phosphorylates TSC2 to block its GTPase activating protein (GAP) activity for Rheb, which activates mTORC1. Amino acids activate mTORC1 through Rag and Vps34/PLD1. Activated mTORC1/S6K1 phosphorylates IRS1 (Ser307 and Ser636/639) and Grb10 to block insulin signaling (negative feedback loop). Overloaded nutrients induce sustained mTORC1 activity that causes insulin resistance. Proteins shown in pink are involved in amino acid mTOR signaling. Proteins shown in green activate insulin-induced Akt activation. PDK1; phosphoinositide-dependent kinase1, mTORC2; mTOR complex2, TSC 2; Tuberous sclerosis complex 2, Rheb; Ras homologue enriched in brain, Vps34; class III PI3 kinase, PLD1; phospholipase D1, IRS1; insulin receptor substrate1, Grb10; growth factor receptor-bound protein 10 . 
inducing IRS1 serine phosphorylation and leading to insulin resistance. $^{44}$

Studies of knockout mice for several mTORC1 components support the role of mTOR in the pathogenesis of type 2 diabetes. Mice that are raptor-deficient in adipose tissue display a lean phenotype and resistance to diet-induced obesity, because of an increase in mitochondrial uncoupling. ${ }^{58}$ Moreover, these mice have improved insulin sensitivity, owing to leanness and the loss of the S6K1-IRS1 negative feedback loop. Consistent with this, S6K1-knockout mice remain sensitive to insulin due to loss of the negative feedback loop, which dampens Ser307 and Ser636/639 phosphorylation of IRS1. ${ }^{49}$ Interestingly, 4EBP1/2-deficient mice have high S6K1 activity, resulting in insulin resistance through S6K1-IRS1. In addition, treatment with rapamycin impedes weight gain in rats and humans in line with these observations. ${ }^{59}$

Notably, the central mTORC1 pathway integrates various metabolic signals including nutrients and hormones, and controls energy homeostasis and body weight. ${ }^{60}$ mTORC1 activity in hyperthalamus is critical for the suppression of feeding by leptin, the adipocyte-derived hormone. Leptin activates hypothalamic mTORC1 and locally injected leucine elevates mTORC1 activity in hypothalamic neurons serving as a redundant signal with leptin, which results in inhibiting both energy intake and body weight gain. However, the effect of rapamycin on food intake and body weight is altered depending on the metabolic context. Rapamycin dampens the anorexigenic effect and weight loss by leptin and cytokine ciliary neurotrophic factor, whereas rapamycin reduced obesity associated with increased mTOR signaling in POMC neuron by aging and POMC-specific TSC deletion. ${ }^{61-63}$ Therefore, both the central mTORC1 pathways and the peripheral mTORC1 pathways are essential to maintaining normal energy and glucose metabolism.

\section{CONCLUDING REMARKS}

Recent findings of new mediators and their regulatory mechanisms have broadened our understanding of amino acid-induced mTOR signaling. In addition to the role of the TSC1-TSC2Rheb hub in transducing upstream signals from growth factors, stressors and energy to mTOR, the lysosomal regulation of mTOR functions as a platform to connect nutrient signals to the Rheb axis. Furthermore, two parallel pathways of amino acid signaling explain the diverse regulation of mTOR signaling. It is yet to be determined which regulators sense amino acids directly and whether the two pathways require separate amino acid sensing mechanisms. The identification of a direct amino acid sensor will shed light on these uncertainties.

A more integrated understanding of mTOR regulation in amino acid signaling will open the door for new therapeutic approaches for metabolic diseases, especially type 2 diabetes. Already, metformin, an antidiabetic drug, inhibits mTOR in an AMP-activated kinase (AMPK)-independent and Ragdependent manner, ${ }^{64}$ providing further support for the idea that the regulation of amino acid sensing could be a therapeutic target for diabetes.

\section{CONFLICT OF INTEREST}

The authors declare no conflict of interest.

\section{ACKNOWLEDGEMENTS}

This work was supported by the Bio \& Medical Technology Development Program of the National Research Foundation (NRF) funded by the Ministry of Science, ICT \& Future Planning (NRF-2014M3A9D5A01073886), by the Korea Healthcare Technology R\&D Project through the Korea Health Industry Development Institute (KHIDI) funded by the Ministry for Health and Welfare, Korea (HI14C1135) and the Gachon University Gil Medical Center (Grant \#2013-33 and 2014-18).

1 Zoncu R, Efeyan A, Sabatini DM. mTOR: from growth signal integration to cancer, diabetes and ageing. Nat Rev Mol Cell Biol 2011; 12: 21-35.

2 Brown EJ, Albers MW, Shin TB, Ichikawa K, Keith CT, Lane WS et al. A mammalian protein targeted by G1-arresting rapamycin-receptor complex. Nature 1994; 369: 756-758.

3 Sarbassov DD, Ali SM, Sabatini DM. Growing roles for the mTOR pathway. Curr Opin Cell Biol 2005; 17: 596-603.

4 Wullschleger S, Loewith R, Hall MN. TOR signaling in growth and metabolism. Cell 2006; 124: 471-484.

5 Pearce LR, Huang X, Boudeau J, Pawlowski R, Wullschleger S, Deak $\mathrm{M}$ et al. Identification of Protor as a novel Rictor-binding component of mTOR complex-2. Biochem J 2007; 405: 513-522.

6 Yang Q, Inoki K, Ikenoue T, Guan KL. Identification of $\operatorname{Sin} 1$ as an essential TORC2 component required for complex formation and kinase activity. Genes Dev 2006; 20: 2820-2832.

7 Frias MA, Thoreen CC, Jaffe JD, Schroder W, Sculley T, Carr SA et al. $\mathrm{mSin} 1$ is necessary for Akt/PKB phosphorylation, and its isoforms define three distinct mTORC2s. Curr Biol 2006; 16: 1865-1870.

$8 \mathrm{Kim}$ DH, Sarbassov DD, Ali SM, Latek RR, Guntur KV, Erdjument-Bromage $\mathrm{H}$ et al. GbetaL, a positive regulator of the rapamycin-sensitive pathway required for the nutrient-sensitive interaction between raptor and mTOR. Mol Cell 2003; 11: 895-904.

9 Sancak Y, Thoreen CC, Peterson TR, Lindquist RA, Kang SA, Spooner $\mathrm{E}$ et al. PRAS40 is an insulin-regulated inhibitor of the mTORC1 protein kinase. Mol Cell 2007; 25: 903-915.

10 Khanna N, Fang Y, Yoon MS, Chen J. XPLN is an endogenous inhibitor of mTORC2. Proc Natl Acad Sci USA 2013; 110: 15979-15984.

11 Bai X, Ma D, Liu A, Shen X, Wang QJ, Liu Y et al. Rheb activates mTOR by antagonizing its endogenous inhibitor, FKBP38. Science 2007; 318: 977-980

12 Peterson TR, Laplante M, Thoreen CC, Sancak Y, Kang SA, Kuehl WM et al. DEPTOR is an mTOR inhibitor frequently overexpressed in multiple myeloma cells and required for their survival. Cell 2009; 137: 873-886.

13 Bar-Peled L, Sabatini DM. Regulation of mTORC1 by amino acids. Trends Cell Biol 2014; 24: 400-406.

$14 \mathrm{Ma}$ L, Chen Z, Erdjument-Bromage H, Tempst P, Pandolfi PP. Phosphorylation and functional inactivation of TSC2 by Erk implications for tuberous sclerosis and cancer pathogenesis. Cell 2005; 121: 179-193.

15 Potter CJ, Pedraza LG, Xu T. Akt regulates growth by directly phosphorylating Tsc2. Nat Cell Biol 2002; 4: 658-665.

16 Inoki K, Zhu T, Guan KL. TSC2 mediates cellular energy response to control cell growth and survival. Cell 2003; 115: 577-590.

17 Garami A, Zwartkruis FJ, Nobukuni T, Joaquin M, Roccio M, Stocker $\mathrm{H}$ et al. Insulin activation of Rheb, a mediator of mTOR/S6K/4EBP signaling, is inhibited by TSC 1 and 2. Mol Cell 2003; 11: 1457-1466.

18 Rui L. A link between protein translation and body weight. J Clin Invest 2007: 117: 310-313.

19 Ma XM, Blenis J. Molecular mechanisms of mTOR-mediated translational control. Nat Rev Mol Cell Biol 2009; 10: 307-318.

20 Hara K, Yonezawa K, Weng QP, Kozlowski MT, Belham C, Avruch J. Amino acid sufficiency and mTOR regulate p70 S6 kinase and elF-4E BP1 through a common effector mechanism. J Biol Chem 1998; 273: 14484-14494. 
21 Wang X, Campbell LE, Miller CM, Proud CG. Amino acid availability regulates p70 S6 kinase and multiple translation factors. Biochem J 1998; 334(Pt 1): 261-267.

22 Avruch J, Hara K, Lin Y, Liu M, Long X, Ortiz-Vega S et al. Insulin and amino-acid regulation of mTOR signaling and kinase activity through the Rheb GTPase. Oncogene 2006; 25: 6361-6372.

23 Nobukuni T, Joaquin M, Roccio M, Dann SG, Kim SY, Gulati P et al. Amino acids mediate mTOR/raptor signaling through activation of class 3 phosphatidylinositol 30H-kinase. Proc Natl Acad Sci USA 2005; 102 : 14238-14243.

24 Binda M, Peli-Gulli MP, Bonfils G, Panchaud N, Urban J, Sturgill TW et al. The Vam6 GEF controls TORC1 by activating the EGO complex. Mol Cell 2009; 35: 563-573.

25 Kim E, Goraksha-Hicks P, Li L, Neufeld TP, Guan KL. Regulation of TORC1 by Rag GTPases in nutrient response. Nat Cell Biol 2008; 10: 935-945.

26 Sancak Y, Peterson TR, Shaul YD, Lindquist RA, Thoreen CC, Bar-Peled L et al. The Rag GTPases bind raptor and mediate amino acid signaling to mTORC1. Science 2008; 320: 1496-1501.

27 Sancak Y, Bar-Peled L, Zoncu R, Markhard AL, Nada S, Sabatini DM. Ragulator-Rag complex targets mTORC1 to the lysosomal surface and is necessary for its activation by amino acids. Cell 2010; 141: 290-303.

28 Bar-Peled L, Schweitzer LD, Zoncu R, Sabatini DM. Ragulator is a GEF for the rag GTPases that signal amino acid levels to mTORC1. Cell 2012; 150: $1196-1208$.

29 Zoncu R, Bar-Peled L, Efeyan A, Wang S, Sancak Y, Sabatini DM. mTORC1 senses lysosomal amino acids through an inside-out mechanism that requires the vacuolar H(+)-ATPase. Science 2011; 334: 678-683.

30 Bar-Peled L, Chantranupong L, Cherniack AD, Chen WW, Ottina KA, Grabiner BC et al. A tumor suppressor complex with GAP activity for the Rag GTPases that signal amino acid sufficiency to mTORC1. Science 2013; 340: 1100-1106.

31 Tsun ZY, Bar-Peled L, Chantranupong L, Zoncu R, Wang T, Kim C et al. The folliculin tumor suppressor is a GAP for the RagC/D GTPases that signal amino acid levels to mTORC1. Mol Cell 2013; 52: 495-505.

32 Bonfils G, Jaquenoud M, Bontron S, Ostrowicz C, Ungermann C, De Virgilio C. Leucyl-tRNA synthetase controls TORC1 via the EGO complex. Mol Cell 2012; 46: 105-110.

33 Han JM, Jeong SJ, Park MC, Kim G, Kwon NH, Kim HK et al. Leucyl-tRNA synthetase is an intracellular leucine sensor for the mTORC1-signaling pathway. Cell 2012; 149: 410-424.

34 Byfield MP, Murray JT, Backer JM. hVps34 is a nutrient-regulated lipid kinase required for activation of p70 S6 kinase. J Biol Chem 2005; 280 33076-33082.

35 Backer JM. The regulation and function of Class III PI3Ks: novel roles for Vps34. Biochem J 2008; 410: 1-17.

36 Sun $\mathrm{Y}$, Fang $\mathrm{Y}$, Yoon MS, Zhang C, Roccio M, Zwartkruis FJ et al. Phospholipase D1 is an effector of Rheb in the mTOR pathway. Proc Natl Acad Sci USA 2008; 105: 8286-8291.

37 Selvy PE, Lavieri RR, Lindsley CW, Brown HA, Phospholipase D. enzymology, functionality, and chemical modulation. Chem Rev 2011; 111: 6064-6119.

38 Fang Y, Vilella-Bach M, Bachmann R, Flanigan A, Chen J. Phosphatidic acid-mediated mitogenic activation of mTOR signaling. Science 2001; 294: 1942-1945.

39 Yoon MS, Sun Y, Arauz E, Jiang Y, Chen J. Phosphatidic acid activates mammalian target of rapamycin complex 1 (mTORC1) kinase by displacing FK506 binding protein 38 (FKBP38) and exerting an allosteric effect. J Biol Chem 2011; 286: 29568-29574.

40 Yoon MS, Rosenberger CL, Wu C, Truong N, Sweedler JV, Chen J. Rapid mitogenic regulation of the mTORC1 inhibitor, DEPTOR, by phosphatidic acid. Mol Cell 2015; 58: 549-556.

41 Sun Y, Chen J. mTOR signaling: PLD takes center stage. Cell Cycle 2008; 7: 3118-3123.

42 Yoon MS, Du G, Backer JM, Frohman MA, Chen J. Class III PI-3-kinase activates phospholipase $\mathrm{D}$ in an amino acid-sensing mTORC1 pathway. J Cell Biol 2011; 195: 435-447.

43 Xu L, Salloum D, Medlin PS, Saqcena M, Yellen P, Perrella B et al. Phospholipase $D$ mediates nutrient input to mammalian target of rapamycin complex 1 (mTORC1). J Biol Chem 2011; 286: 25477-25486.

44 Newgard CB, An J, Bain JR, Muehlbauer MJ, Stevens RD, Lien LF et al. A branched-chain amino acid-related metabolic signature that differentiates obese and lean humans and contributes to insulin resistance. Cell Metab 2009; 9: 311-326.

45 McCormack SE, Shaham O, McCarthy MA, Deik AA, Wang TJ, Gerszten RE et al. Circulating branched-chain amino acid concentrations are associated with obesity and future insulin resistance in children and adolescents. Pediatr Obes 2013; 8: 52-61.

46 Lackey DE, Lynch CJ, Olson KC, Mostaedi R, Ali M, Smith WH et al. Regulation of adipose branched-chain amino acid catabolism enzyme expression and cross-adipose amino acid flux in human obesity. Am J Physiol Endocrinol Metab 2013; 304: E1175-E1187.

47 Badoud F, Lam KP, DiBattista A, Perreault M, Zulyniak MA, Cattrysse $B$ et al. Serum and adipose tissue amino acid homeostasis in the metabolically healthy obese. J Proteome Res 2014; 13: 3455-3466.

48 Wang TJ, Larson MG, Vasan RS, Cheng S, Rhee EP, McCabe E et al. Metabolite profiles and the risk of developing diabetes. Nat Med 2011; 17: 448-453.

49 Um SH, Frigerio F, Watanabe M, Picard F, Joaquin M, Sticker M et al. Absence of S6K1 protects against age- and diet-induced obesity while enhancing insulin sensitivity. Nature 2004; 431: 200-205.

50 Tremblay $\mathrm{F}$, Jacques $\mathrm{H}$, Marette $\mathrm{A}$. Modulation of insulin action by dietary proteins and amino acids: role of the mammalian target of rapamycin nutrient sensing pathway. Curr Opin Clin Nutr Metab Care 2005; 8: 457-462.

$51 \mathrm{Um} \mathrm{SH}$, D'Alessio D, Thomas G. Nutrient overload, insulin resistance, and ribosomal protein S6 kinase 1, S6K1. Cell Metab 2006; 3: 393-402.

52 Yu Y, Yoon So, Poulogiannis G, Yang Q, Ma XM, Villen J et al. Phosphoproteomic analysis identifies Grb10 as an mTORC1 substrate that negatively regulates insulin signaling. Science 2011; 332: 1322-1326.

53 Hsu PP, Kang SA, Rameseder J, Zhang Y, Ottina KA, Lim D et al. The mTOR-regulated phosphoproteome reveals a mechanism of mTORC1mediated inhibition of growth factor signaling. Science 2011; 332: $1317-1322$

54 Yea SS, Fruman DA. Cell signaling. New mTOR targets Grb attention. Science 2011; 332: 1270-1271.

55 Olson KC, Chen G, Xu Y, Hajnal A, Lynch CJ. Alloisoleucine differentiates the branched-chain aminoacidemia of Zucker and dietary obese rats. Obesity (Silver Spring) 2014; 22: 1212-1215.

56 Kim SG, Hoffman GR, Poulogiannis G, Buel GR, Jang YJ, Lee KW et al. Metabolic stress controls mTORC1 lysosomal localization and dimerization by regulating the TTT-RUVBL1/2 complex. Mol Cell 2013; 49: 172-185.

57 Nicklin $\mathrm{P}$, Bergman $\mathrm{P}$, Zhang B, Triantafellow E, Wang H, Nyfeler B et al. Bidirectional transport of amino acids regulates mTOR and autophagy. Cell 2009; 136: 521-534.

58 Polak P, Cybulski N, Feige JN, Auwerx J, Ruegg MA, Hall MN. Adipose-specific knockout of raptor results in lean mice with enhanced mitochondrial respiration. Cell Metab 2008; 8: 399-410.

59 Rovira J, Marcelo Arellano E, Burke JT, Brault Y, Moya-Rull D, Banon-Maneus $\mathrm{E}$ et al. Effect of mTOR inhibitor on body weight: from an experimental rat model to human transplant patients. Transpl Int 2008; 21: 992-998.

60 Cota D, Proulx K, Smith KA, Kozma SC, Thomas G, Woods SC et al. Hypothalamic mTOR signaling regulates food intake. Science 2006; 312 : 927-930.

61 Yang SB, Tien AC, Boddupalli G, Xu AW, Jan YN, Jan LY. Rapamycin ameliorates age-dependent obesity associated with increased mTOR signaling in hypothalamic POMC neurons. Neuron 2012; 75: 425-436.

62 Mori $\mathrm{H}$, Inoki K, Munzberg H, Opland D, Faouzi M, Villanueva EC et al. Critical role for hypothalamic mTOR activity in energy balance. Cell Metab 2009; 9: 362-374.

63 Cota D, Matter EK, Woods SC, Seeley RJ. The role of hypothalamic mammalian target of rapamycin complex 1 signaling in diet-induced obesity. J Neurosci 2008; 28: 7202-7208.

64 Kalender A, Selvaraj A, Kim SY, Gulati P, Brule S, Viollet B et al. Metformin, independent of AMPK, inhibits mTORC1 in a rag GTPasedependent manner. Cell Metab 2010; 11: 390-401.

(1) () $\Theta$ This work is licensed under a Creative Commons Attribution-NonCommercial-NoDerivs 4.0 International License. The images or other third party material in this article are included in the article's Creative Commons license, unless indicated otherwise in the credit line; if the material is not included under the Creative Commons license, users will need to obtain permission from the license holder to reproduce the material. To view a copy of this license, visit http://creativecommons.org/licenses/by-nc-nd/4.0/ 